\title{
The inner halo of M 87: a first direct view of the red-giant population
}

\author{
S. Bird ${ }^{1}$, W. E. Harris ${ }^{2}$, J. P. Blakeslee ${ }^{3}$, and C. Flynn ${ }^{1,4,5}$ \\ 1 Tuorla Observatory, University of Turku, Väsiäläntie 20, 21500 Piikkiö, Finland \\ e-mail: [sarbir;cflynn] @utu.fi \\ 2 Department of Physics and Astronomy, McMaster University, Hamilton L8S 4M1, Canada \\ e-mail: harris@physics.mcmaster.ca \\ ${ }^{3}$ Herzberg Institute of Astrophysics, National Research Council of Canada, Victoria BC V9E 2E7, Canada \\ e-mail: john.blakeslee@nrc.ca \\ 4 Finnish Centre for Astronomy with ESO (FINCA), University of Turku, Väsiäläntie 20, 21500 Piikkiö, Finland \\ 5 Department of Physics and Astronomy, University of Sydney, Sydney, Australia
}

Received 27 April 2010 / Accepted 15 September 2010

\begin{abstract}
An unusually deep $(V, I)$ imaging dataset for the Virgo supergiant M 87 with the Hubble Space Telescope ACS successfully resolves its brightest red-giant stars, reaching $M_{I}(\mathrm{lim})=-2.5$. After assessing the photometric completeness and biasses, we use this material to estimate the metallicity distribution for the inner halo of M 87, finding that the distribution is very broad and likely to peak near $[m / \mathrm{H}] \simeq-0.4$ and perhaps higher. The shape of the MDF strongly resembles that of the inner halo for the nearby giant $\mathrm{E}$ galaxy NGC 5128. As a byproduct of our study, we also obtain a preliminary measurement of the distance to M 87 with the TRGB (red-giant branch tip) method; the result is $(m-M)_{0}=31.12 \pm 0.14(d=16.7 \pm 0.9 \mathrm{Mpc})$. Averaging this result with three other recent techniques give a weighted mean $d(M 87)=(16.4 \pm 0.5) \mathrm{Mpc}$.
\end{abstract}

Key words. galaxies: stellar content - galaxies: halos - galaxies: individual: M 87

\section{Introduction}

A key route to understanding the early enrichment history of large galaxies is through direct, star-by-star measurement of the metallicity distribution function (MDF) of its halo stellar population. Direct resolution of the old-halo stars with ground-based imaging is, however, generally restricted to the relatively small number of galaxies in the Local Group and not much beyond. The Hubble Space Telescope cameras with their superior spatial resolution and depth are able to extend this type of study considerably further, bringing many more galaxies of all types within reach.

The rarest and biggest types of galaxies are the giant elliptical (gE) types, and to date, very few direct measurements of their MDFs have been made through their halo-star populations. Data of this type have been obtained with HST imaging only for the nearest gE galaxies, namely NGC 5128 at $d=3.8 \pm 0.1 \mathrm{Mpc}$ (Harris et al. 1999, 2000, 2002, 2010; Rejkuba et al. 2004) and NGC 3379 at $d=10.5 \pm 0.8 \mathrm{Mpc}$ (Gregg et al. 2004; Harris et al. $2007 b$ ). To these we may add the MDF study of the intermediateluminosity Leo elliptical NGC 3377, at $d=10.8 \pm 0.5 \mathrm{Mpc}$ (Harris et al. 2007a). Mouhcine et al. (2005b, 2007) have used HST images to directly measure the halo-star MDF of nine spiral galaxies, finding a correlation between overall luminosity and the mean halo metallicity, and suggest that halos of massive spirals may not be dominated by metal weak stars. For this kind of work, the Virgo cluster of galaxies at $d \simeq 16 \mathrm{Mpc}$ is an especially tantalizing arena because it holds a rich collection of all types of galaxies including many large ellipticals. Among all of the Virgo members, however, M 87 (NGC 4486) can be claimed to be the most intriguing target because it is the nearest example we have of a centrally dominant cD-type galaxy, which is likely according to the current galaxy formation framework (e.g. Thomas et al. 2005; Renzini 2006, for comprehensive discussions) to have undergone its major star-forming period very quickly in the early universe.

In this paper, we take advantage of an unusual dataset in the HST Archive to probe the inner-halo stellar population of M 87 - that is, its fundamental population of old red-giant stars - for the first time. As will be seen in the discussion below, our ability to gauge the MDF accurately is affected by the high level of photometric crowding in the images. Despite its limitations, however, it is good enough to permit some preliminary steps towards measuring the stellar MDF. In the following sections, we describe the raw data and our preprocessing; the photometric methods; measurement of the red-giant-branch tip and distance calibration; and finally a preliminary discussion of the metallicity distribution function.

\section{The dataset and preliminary processing}

The raw images we use in this study are from HST program GO-10543 (PI Baltz) and were taken with the ACS (Advanced Camera for Surveys) Wide Field Channel in the $F 606 W$ (wide $V$ ) and $F 814 W$ (wide $I$ ) filters. The $3.4^{\prime} \times 3.4^{\prime}$ field, corresponding to $16 \times 16 \mathrm{kpc}$ linear scale at the Virgo distance, was centered on M 87. The exposures were taken in 61 separate spacecraft visits, extending over a 71-day period from 2005 Dec. 24 to 2006 March 5. These many exposures were originally designed to search for microlensing events. In $F 814 W$ there are 205 images totalling 73800 s, while in $F 606 W$ there are 49 images totalling $24500 \mathrm{~s}$. Co-adding the individual exposures in each filter yields an unusually deep pair of images at higher scale and a certain amount of gain in spatial resolution. 
Composite exposures of these images have already been used for three separate studies of the population of $\simeq 2000$ globular clusters that lie within the area of this ACS pointing (Waters et al. 2009; Peng et al. 2009; Madrid et al. 2009).

If the distance to M 87 is $16 \mathrm{Mpc}$ (see below), the expected magnitude of the TRGB (red-giant branch tip) at $M_{I}=-4.05$ (Rizzi et al. 2007) is $I_{\mathrm{TRGB}} \simeq 27.0$. As will be seen below, the limiting $V, I$ magnitudes of these co-added exposures are easily capable of directly resolving the TRGB and delineating the color-magnitude distribution (CMD) of the brightest RGB stars. The CMD can, in turn, be used to derive the MDF, as well as a direct calibration of the distance to M 87 through the luminosity of the RGB tip.

For the purposes of this study, we carried out a new combination of all the raw exposures in each filter through the APSIS software (Blakeslee et al. 2003), which performs accurate image registration, cosmic-ray rejection, and distortion correction with drizzle. Many hundreds of sources were used for measuring the offsets and small rotations of the individual exposures, yielding an accuracy of the registration of the raw images better than $0.01 \mathrm{px}$. The unusually large numbers of exposures in each filter permitted subpixel resampling; we chose to construct final combined science images with a scale $0.025 \mathrm{px}^{-1}$ (half the native pixel size of the camera), and used the drizzle "point" (deltafunction) interpolation kernel, which is equivalent to a square kernel with a pixfrac parameter of zero. Note that in the previous globular cluster analyses of Peng et al. (2009) and Madrid et al. (2009) we generated combined images with the Gaussian kernel and a scale of 0 . $^{\prime} 035 \mathrm{px}^{-1}$. However, for the present study, we decided to rebuild the final science frames to gain the best possible effective resolution for faint-star photometry.

A portion of our final science image in $F 814 W$, illustrating the degree of crowding in an outer corner of the field, is shown in Fig. 1. The excised sample shown in the figure is located in the southeast corner of the ACS field, at $1230^{\prime} 58.576^{\prime \prime}$ and $+12^{\circ} 24^{\prime} 39.00^{\prime \prime}$, at a projected galactocentric distance of about $150^{\prime \prime}$ or $12.1 \mathrm{kpc}$. The mean surface brightness of the region in Fig. 1 in $V$ and $I$ bands is $\mu_{V}=22.4 \mathrm{mag} / \mathrm{sq} \operatorname{arcsec}$ and $\mu_{I}=$ $21.2 \mathrm{mag} / \mathrm{sq}$ arcsec, from the surface photometry of Kormendy et al. 2009 (see also Zeilinger et al. 1993). The location of this field is shown in Fig. 2.

Another similarly deep probe of the RGB population in the Virgo region has been published by Williams et al. (2007). Their HST/ACS target field (from program GO-10131) is located in the intracluster region between M 87 and M 86, approximately $180 \mathrm{kpc}$ projected distance from each of these two giants. Their data consist of $26880 \mathrm{~s}$ exposure time in $F 814 \mathrm{~W}$ and $63440 \mathrm{~s}$ in $F 606 \mathrm{~W}$, very similar total integration but with the opposite color emphasis than our central M 87 field has. As Williams et al. discuss, the stellar population in this intracluster field is predominantly metal-poor, and is likely to have a large mixture of material tidally stripped from many Virgo members. By contrast, the M 87-centered field we analyze here should provide a relatively pure sample of the inner-halo RGB stars in this supergiant $E$ alone and can be expected to be more metal-rich on average.

\section{Photometry}

To define the point-spread function (PSF) empirically for stellar photometry, we selected 22 moderately bright, isolated stars located across the ACS field and used these same stars for both filters (only about 40 bright stars in total are clearly distinguishable in the entire field among the 2000 globular clusters; see Madrid et al. 2009). Our target RGB halo stars are far fainter than these

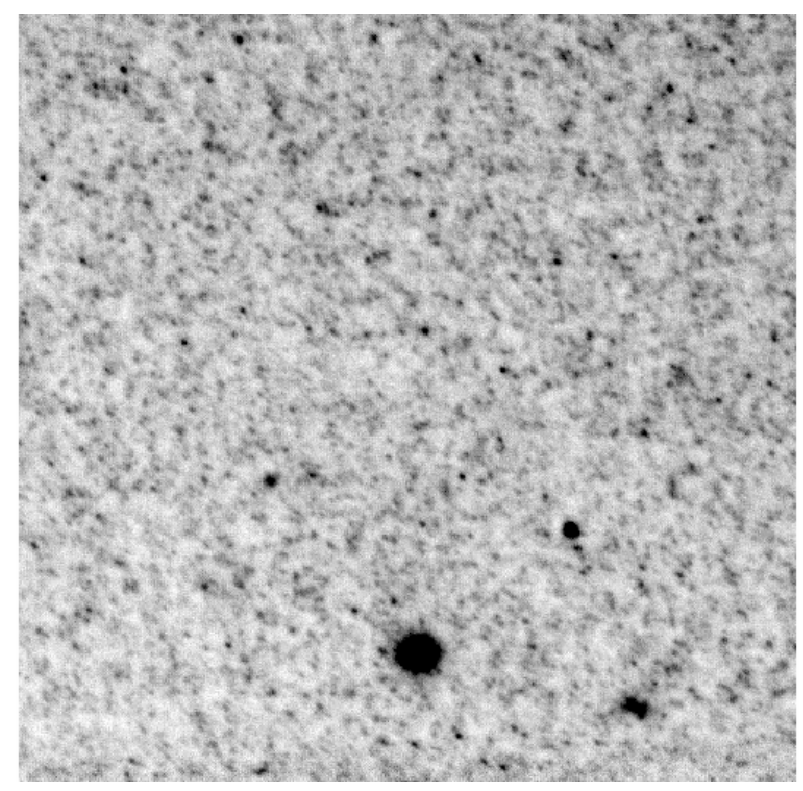

Fig. 1. A $12.5^{\prime \prime} \times 12.5^{\prime \prime}$ portion of the composite $F 814 \mathrm{~W}$ image of the M 87 halo, demonstrating its resolution into stars. Three globular clusters are seen in the lower half of the picture. This section is located in the extreme southeast corner of the ACS field, $\simeq 150^{\prime \prime}$ or $12.1 \mathrm{kpc}$ from the center of M 87. Stellar crowding increases rapidly toward the galaxy center but is manageable at this location.

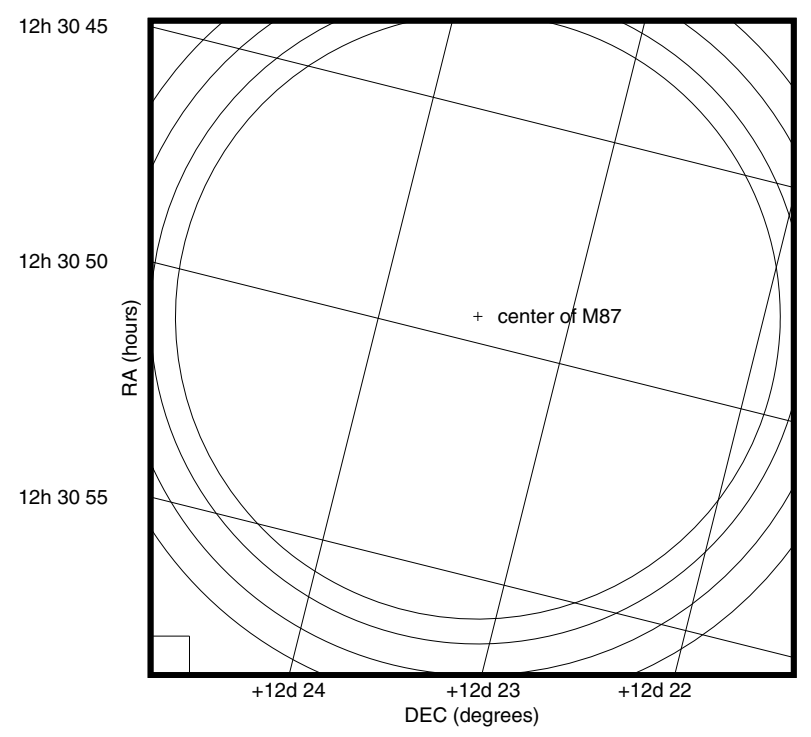

Fig. 2. Full ACS field of view. Lines corresponding to declination versus right ascension are drawn. Four annuli mark regions used in the photometry at $97^{\prime \prime}-105^{\prime \prime}, 105^{\prime \prime}-115^{\prime \prime}, 115^{\prime \prime}-125^{\prime \prime}$, and $125^{\prime \prime}-155^{\prime \prime}$, and are drawn around the center of M 87. Note that the 155" circle does not show, because it is coincident with the bottom left corner of the ACS image. The central circle and the first two annuli are not used for final analysis due to crowding. Fig. 1 is the portion marked by a square in the lower left corner.

PSF stars, and the internal measurement uncertainties are dominated completely by sky noise and crowding, so the fine details of the PSF shape are not critical for this purpose. Nevertheless, we found no detectable differences in PSF size as a function of location in the field after the APSIS distortion corrections (see also Madrid et al. for further discussion). The $F W H M$ of the PSF we obtained is $3.2 \mathrm{px}\left(0.080^{\prime \prime}\right)$ in $F 606 W$ and $3.4 \mathrm{px}\left(0.085^{\prime \prime}\right)$ in $F 814 W$, yielding a small but useful gain in effective resolution 
S. Bird et al.: The inner halo of M 87: a first direct view of the red-giant population

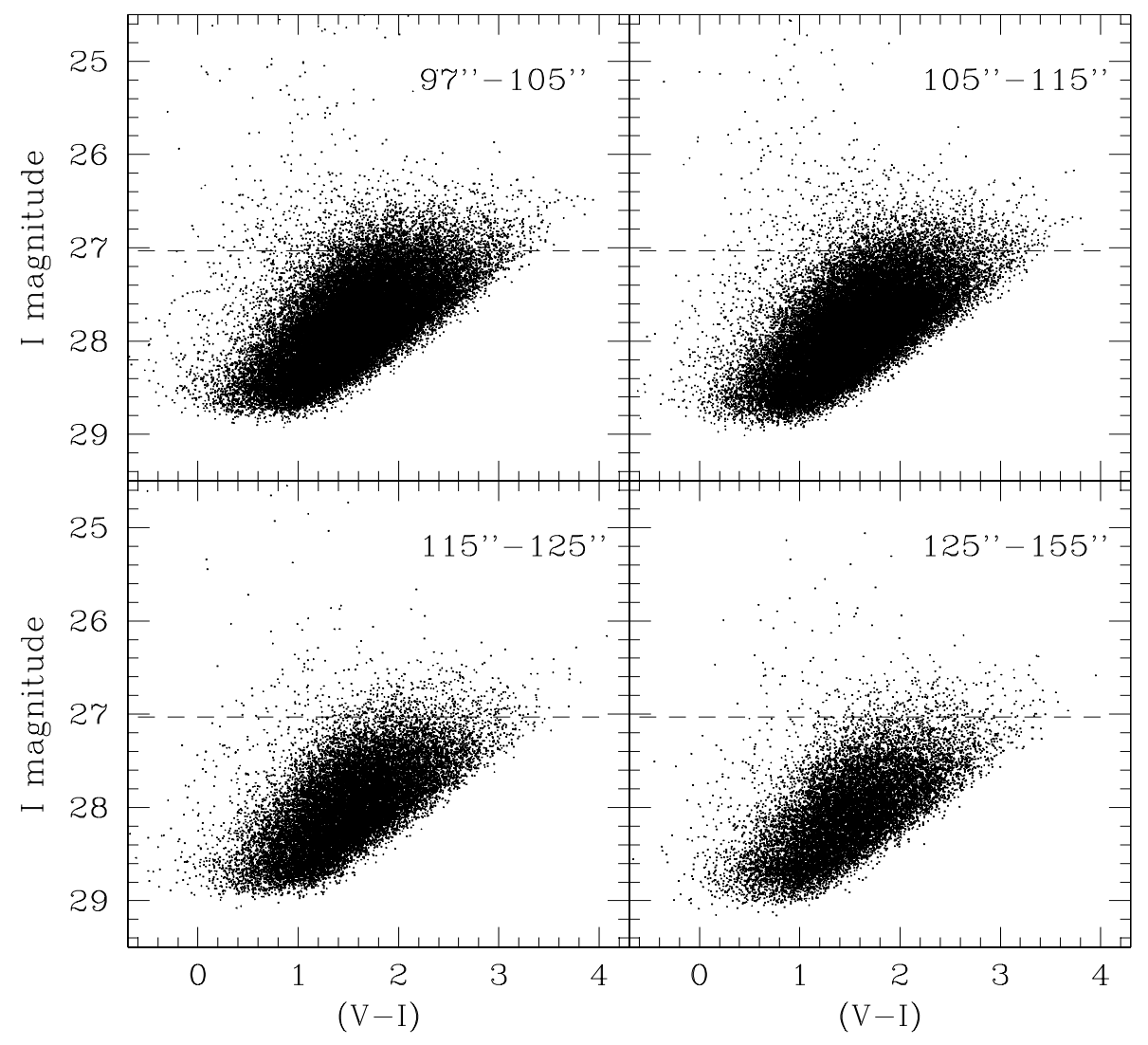

Fig. 3. The color-magnitude distribution ( $I$ vs. $(V-I))$ for the measured stars in the M 87 inner halo. Data are plotted in four concentric annuli in projected galactocentric distance. These annuli cover a total radial range in $R_{\mathrm{gc}}$ from $7.9 \mathrm{kpc}$ to $12.5 \mathrm{kpc}$. The horizontal dashed line in each panel shows the predicted level of the RGB tip at $M_{I}=-4.05$ (see text). compared with the nominal $0.1^{\prime \prime}$ resolution of the individual exposures.

Across almost all the area of this M 87-centered ACS field the level of stellar crowding is, to say the least, severe. It is only in the outermost corners that crowding is low enough to permit star-by-star identification and photometry by normal techniques. Once the PSF was established, we masked off all the area within 3900 px (7.9 kpc or 97") projected galactocentric distance, essentially leaving only the four corners. This outer measured region is marked in Fig. 2. To carry out the photometry, we used the IRAF daophot codes (see Stetson 1987) with a 2-pass sequence of find/phot/allstar. For allstar the PSF fitting radius was adopted as $1.5 \mathrm{px}$ for the final runs to reduce crowding effects as much as possible, though we found that slightly larger values up to 2.5 px did not give significantly different results. Correction of the PSF-fitted instrumental magnitudes to large-aperture radius was done by curve-of-growth measurements from the same set of bright stars that were used to define the PSF. Lastly, these aperture-corrected instrumental magnitudes were converted to $F 606 W, F 814 W$ magnitudes and then into $(V, I)$ with the current ACS/WFC filter zeropoints and conversion relations in the VEGAmag system (see Sirianni et al. 2003 and the ACS web pages $^{1}$ ),

$$
\begin{aligned}
F 606 W & =-2.5 \log \left(f_{V}\right)+26.421 \\
F 814 W & =-2.5 \log \left(f_{I}\right)+25.536 \\
V & =F 606 W+0.236(V-I) \\
I & =F 814 W-0.002(V-I)
\end{aligned}
$$

where $f_{V}, f_{I}$ are the flux rates in counts per second for largeaperture photometry. The dominant uncertainty in the calibration is in the correction from the small radii $(2 \mathrm{px})$ that we are

\footnotetext{
${ }^{1}$ http://www.stsci.edu/hst/acs/analysis/zeropoints
}

forced to use because of the high degree of crowding, to "infinite" large-aperture radius through the curves of growth. From these we believe the true external uncertainty in the photometric zeropoints to be $\pm 0.1 \mathrm{mag}$ in each filter.

Over the outer region defined above, our final data consist of 105466 measured stars. The color-magnitude array for these is shown in Fig. 3, subdivided into the four annular zones that are shown in Fig. 2. To facilitate comparison between zones, the expected level of the RGB tip luminosity for metal-poor $([\mathrm{Fe} / \mathrm{H}] \lesssim-0.7) \mathrm{RGB}$ stars at $M_{I}=-4.05$ (Rizzi et al. 2007) is shown in each panel as the dashed line. For our present purposes, to minimize crowding effects as much as possible we restrict our following discussion to the two outermost zones $\left(R_{\mathrm{gc}}=115^{\prime \prime}-155^{\prime \prime}\right)$, containing 33890 measured stars.

The RGB spans a broad color range, suggesting a large range in metallicity, or very large internal photometric scatter, or both. Our ability to see the true metallicity spread of the RGB tip is limited by the $F 606 \mathrm{~W}$ exposures, which set the very distinct red-edge cutoff to the data at $V \lesssim 30$ (for similar cases, see the NGC 3379 or NGC 5128 studies of Harris et al. 2002, 2007b where the most metal-rich part of the population is cut off). A more specific demonstration of this point is shown in Fig. 4, where the combined photometry for the two outermost annuli is plotted along with fiducial tracks for 12-Gyr-old RGB stars over the metallicity range $[\mathrm{Fe} / \mathrm{H}]=-2.3$ to +0.4 . These tracks are the same ones used in previous studies of NGC 5128 and NGC 3379 (Harris et al. 2002, 2007b) and are drawn primarily from the model library of VandenBerg et al. (2000). At the right-hand edge of the CMD, the detection limits set by the $V$ filter prevent us from measuring any stars more metal-rich than $[m / H] \simeq-0.2$ (i.e. stars in the range of the three reddest tracks in the model grid). In addition, at levels $I \gtrsim 27.6$ the increasing photometric measurement uncertainties produce an asymmetric scatter in the 
observed colors: objects measured too blue by random errors fall well to the blue side of the most metal-poor model track; contrarily, any that are measured too red by similar amounts fall below the $V$ limits and do not appear on the CMD. These arguments suggest that the MDF derivation needs to be restricted to only the very brightest section of the RGB to minimize biasses and excessive random spread. We will quantify this approach below after a brief discussion of the TRGB and distance calibration.

\section{Determining the TRGB and fiducial distance}

Although the main focus of our M 87 study is the metallicity distribution, the data are also suitable for a preliminary estimate of the tip of the red-giant branch (TRGB). The bright-end termination of the red giant branch has proven to be an extremely effective standard candle for distance measurement to nearby galaxies (well reviewed by Rizzi et al. 2007). Observationally it has the strong advantages that only single-epoch imaging is needed; that it is used for halo stars where internal differential reddening is usually not an issue; and that it rests soundly on a well defined and well understood breakpoint in the evolution of old, low-mass stars that are universally present in most galaxies. In addition, it is most easily employed in the $I$ band where the level of the TRGB is nearly flat for the generous metallicity range $[\mathrm{Fe} / \mathrm{H}] \lesssim-0.7$ (see Rizzi et al. and papers cited there).

The TRGB, which physically represents the helium-ignition point along the evolutionary tracks of the red-giant stars, is empirically defined by a sudden rise in the luminosity function (LF) of the halo stars. For recent examples of the sharp, near-ideal TRGBs that result from uncrowded photometry and statistically large samples of stars, see McConnachie et al. (2005), Mouhcine et al. (2005a), or Harris et al. (2007b). However, our M 87 photometry is unavoidably in a different regime: because of the crowding and substantial star-by-star photometric uncertainties (which are already as large as $\pm 0.36 \mathrm{mag}$ at the top of the RGB, as detailed in the next section), the TRGB does not appear here as a sharp onset in the LF but rather a smoothed-out rise. In Fig. 5 we show the LF for the measured stars in the outer radial range $\left(115^{\prime \prime}-155^{\prime \prime}\right)$ of our M 87 ACS field. Here, the data are smoothed with a Gaussian kernel of $\sigma=0.01 \mathrm{mag}$, though the result is insensitive to the particular smoothing width. The numerical first derivative $(\mathrm{d} n / \mathrm{d} I)$ and second derivative $\left(\mathrm{d}^{2} n / \mathrm{d} I^{2}\right)$ or "edge response filter" ERF are shown below the smoothed LF.

A prominent increase in the ERF amplitude first appears at $I=26.98 \pm 0.03$ (internal uncertainty), which we identify as the TRGB. This apparent magnitude must be adjusted for a systematic photometric error (see next section) of $\Delta I=$ $0.12 \mathrm{mag}$, yielding a true TRGB level $I(T R G B)=27.10$. Adopting $M_{I}(T R G B)=-4.05 \pm 0.10$ (Rizzi et al. 2007), we obtain an apparent distance modulus $(m-M)_{I}=31.15 \pm 0.14$, where we have now included the \pm 0.1 - mag uncertainty in the photometric zeropoint (see above). Subtracting $A_{I}=0.03$ as listed in the NED then gives $(m-M)_{0}=31.12 \pm 0.14$ and a true distance to $\mathrm{M} 87$ of $d=(16.7 \pm 0.9) \mathrm{Mpc}$.

As a secondary test of the degree to which crowding alone affects our result, we show in Fig. 6 the smoothed LF and ERF for the four separate radial zones defined in Figs. 2 and 3. Aside from differences in the total numbers of stars, no significant offsets are seen in the estimated TRGB from field to field.

Given that the ERF technique is difficult to apply in such high crowding regimes, we estimated the TRGB level with an independent approach, similar to the ones used by Harris et al. (1998) and McConnachie et al. (2004). Here, the LF of the observed stars is modelled as the sum of an AGB and RGB for an

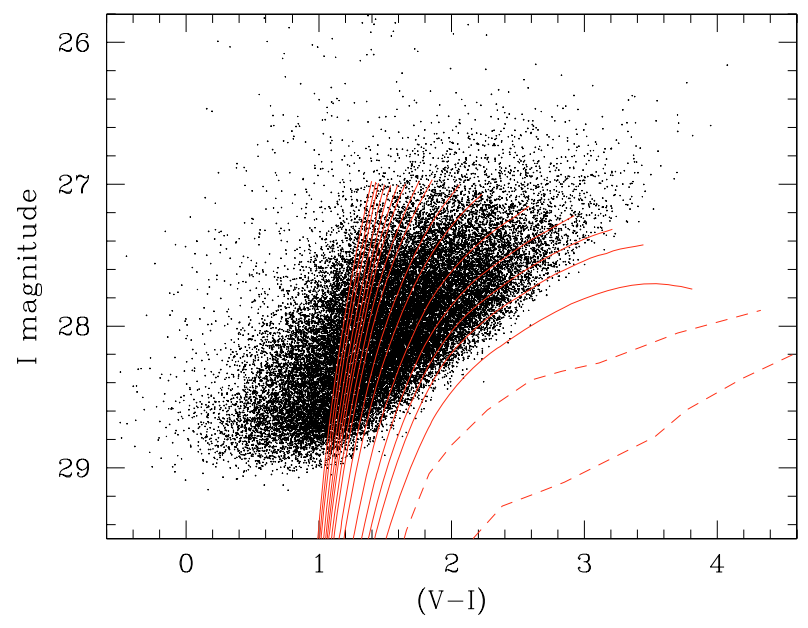

Fig. 4. Color-magnitude distribution $(I$ vs. $(V-I))$ for the measured stars in the outer radial range $\left(115^{\prime \prime}-155^{\prime \prime}\right)$ of our ACS field. The superimposed solid lines are fiducial 12-Gyr RGB tracks from the VandenBerg et al. 2000 model library, in steps of roughly 0.1 dex from $\log \left(Z / Z_{\odot}\right)=-2.0$ to -0.1 . These are supplemented with empirical tracks for two high-metallicity values at $\log \left(Z / Z_{\odot}\right)=+0.16$ and +0.4 (dashed lines; see Harris et al. 2002).
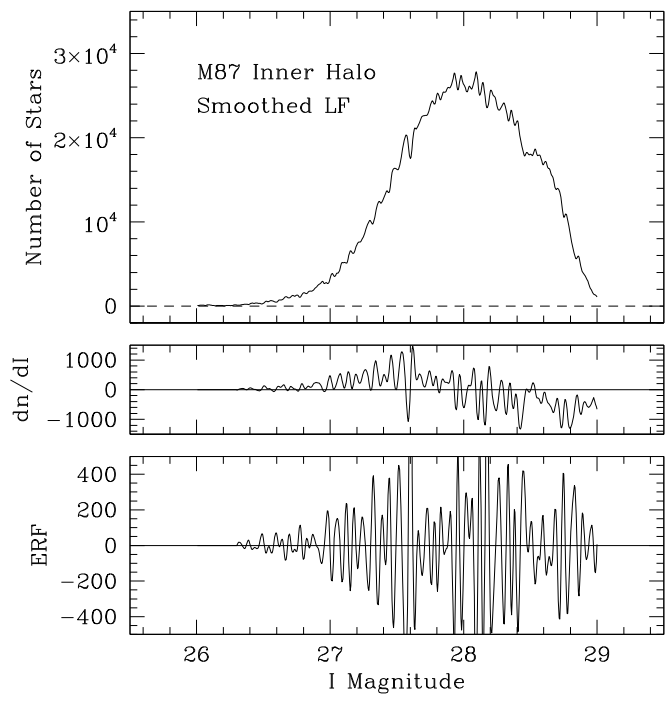

Fig. 5. Definition of the luminosity function for the M 87 stars and the TRGB. Top panel shows all stars in the outer radial range $\left(115^{\prime \prime}-155^{\prime \prime}\right)$ of our ACS field, smoothed with a 0.01-mag Gaussian kernel. Middle and bottom panels show the numerically derived first and second derivatives of the LF.

adopted distance modulus, convolved with the photometric errors, and compared to the observed LF. The RGB is assumed to have a step function onset at the TRGB, rising exponentially to fainter magnitudes; the AGB is assumed to start one magnitude brighter than the TRGB, rising exponentially but with lower amplitude than the RGB. (In practice, the AGB can be thought of as the sum of the true old-AGB stars plus blended pairs from the RGB). The exponential slopes are set to $\Delta \log N / \Delta I=0.96$, and the normalisation of the AGB to the RGB is a free parameter. We convolve this model by the photometric error and completeness as functions of $I$ magnitude (determined from our tests with artificial stars), and compare it to the observed LF. We found good fits to the observed LF for an M 87 TRGB at $I=27.1 \pm 0.2$ and an AGB-to-RGB ratio at the TRGB of $\approx 5-10$ percent. The AGB/RGB ratio plays only a minor role in 
the fitting, which is most sensitive to I(TRGB). The fitting corroborates $I($ TRGB $)=27.1$ derived from the ERF method above.

The stars lying above the RGB tip will be a combination of (a) field contamination from both foreground stars and faint, small background galaxies; (b) stars that are genuinely part of the M 87 halo/bulge population, either young evolved stars or old AGB stars in highly evolved states including long-period variables; and (c) accidental blends of two or more normal RGB stars. The field contamination component $N_{f}$ is independent of the number $N_{\mathrm{RGB}}$ of M 87 stars; the number of AGB stars goes in proportion to $N_{\mathrm{RGB}}$; and the number of blends varies as $N_{\mathrm{RGB}}^{2}$. For our two outer zones, there are 761 stars in the 0.7 -mag region just above the TRGB, versus 4467 stars in the 0.5-mag region just below it. For field contamination, comparison with the photometry of Williams et al. (2007) and Madrid et al. (2009) suggests that $\$ 40$ foreground stars are present, a negligible contribution. The number of faint, small and misidentified background galaxies is harder to assess without an adjacent control field to rely on, but comparison with the similar $\operatorname{ACS}(V, I)$ Leo field data of Harris et al. (2007a) suggests very conservatively $N_{\text {gal }} \lesssim 50$, also negligible.

For the NGC 3379 halo field, Harris et al. (2007b) found that the number of LPVs was $2.5 \%$ of the number of stars in the brightest $0.5 \mathrm{mag}$ of the RGB. Scaling from that fraction then indicates we should expect $N_{\mathrm{LPV}} \simeq 110$ in our two outer zones. These plus the field contaminants then account for about a quarter of the CMD stars above the TRGB. There is no evidence for any young population of stars, which if present would produce an upward extension of the blue side of the RGB (see Williams et al. 2007, for an example).

Most or all of the remainder is likely to be due to blends: we expect (see Harris et al. 2007a)

$N_{\text {blend }} \simeq \frac{N_{\star}^{2}}{2} \frac{a}{A}$

where $N_{\star}$ is the number of stars in the field capable of generating a blended pair brighter than the TRGB, $a$ is the area of one resolution element, and $A$ is the area of the field. We take $A=1.42 \operatorname{arcmin}^{2}$ for the area of the outer two annuli enclosed within the field; $a=7.8 \times 10^{-3} \operatorname{arcsec}^{2}$; and $N_{\star} \simeq 16000$ for the number of stars between $I=27$ and 28 , the top magnitude of the RGB. These parameters predict about 200 blends lying above the TRGB. In fact this estimate is only a lower limit, because (as discussed in the next section) crowding-driven incompleteness of the photometry affects even the range $I<28$ and $N_{\star}$ could be quite a bit larger than our directly measured count. Though admittedly provisional, our conclusion is that the great majority of the objects brighter than the TRGB can be accounted for by the combination of the three normal factors given above.

One useful comparison study of the distance to M 87 is the photometry of the intracluster stars in Virgo by Williams et al. (2007). Although their target field is far from M 87, it should in principle have a reasonably similar TRGB level given that M 87 is near the physical center of the Virgo environment. Although they do not specifically derive the TRGB, their CMD for the intracluster stars clearly shows $I(\mathrm{TRGB}) \simeq 26.9-27.0$, close to our value for M 87 itself.

Other indirect, but still quite relevant, TRGB-based calibrations of the Virgo distance include studies of a small number of its dwarf ellipticals. Harris et al. (1998) obtained $(m-M)_{0}=$ $30.98 \pm 0.20$ for VCC1104 = IC3388, while Caldwell (2006) obtained $(m-M)_{0}=31.03 \pm 0.1$ for seven other dwarfs. All of these are consistent with membership in the Virgo cluster, and with our result for $\mathrm{M} 87$.
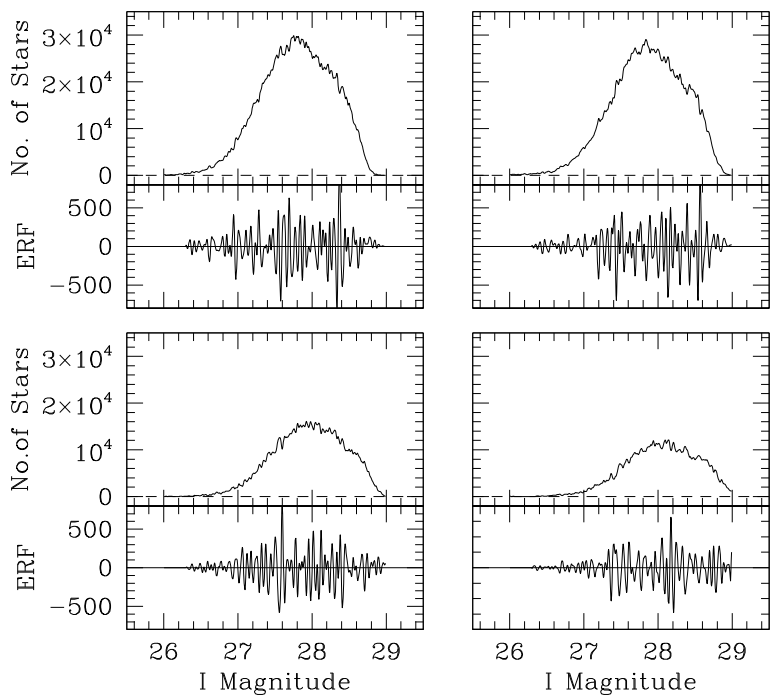

Fig. 6. The luminosity function and edge response filter calculations for the four radial zones defined in Fig. 3 above. All have been determined in the same way as in the previous figure.

The only other direct distance calibrations for M 87 that rely on resolved stars are: (a) the observations of two novae (Pritchet $\&$ van den Bergh 1985), from which they obtain only the extremely uncertain range of 30.4-32.4 for the distance modulus; and (b) the planetary nebula luminosity function (PNLF) by Ciardullo et al. (1998). After adjustment of their adopted zeropoint $M^{\star}(\lambda 5007)=-4.54$ upward to $M^{\star}=-4.67$ to reflect a change in the fiducial M 31 distance (see Harris et al. 2010), the PNLF modulus is $(m-M)_{0}=30.92 \pm 0.16$. The modulus is 0.2 mag smaller than our TRGB value, though the two methods agree within their combined uncertainties.

The largest and most precise compilation of relative distances for the Virgo galaxies is by Blakeslee et al. (2009; see also Mei et al. 2007), from the surface brightness fluctuations (SBF) technique. SBF represents a "secondary" standard-candle method because it relies for its calibration on nearby galaxies whose distances are known beforehand from resolved-star methods, although theoretical calibration via stellar population models is also possible. Blakeslee et al. (2009) determine $(m-M)_{0}=$ $31.11 \pm 0.08$ for M 87 in particular, starting from a mean distance modulus of $31.09 \pm 0.03$ for the Virgo cluster as a whole. This in turn is based on the Cepheid calibration of the SBF method given by Tonry et al. (2001), corrected following Blakeslee et al. (2002) to the final set of Key Project Cepheid distances (Freedman et al. 2001).

Our TRGB distance measurement, and the earlier PNLF measurement, are gauges of the M 87 distance that rely much less on Cepheids. The PNLF zeropoint, for example, relies more heavily on the fiducial distance to M 31, for which close agreement now holds among several standard candles including RR Lyrae stars, TRGB, and Cepheids. Insofar as all these methods start from a common, fundamental Local Group distance scale that has been carefully assembled by a combination of many standard candles, the $\sim \pm 0.2$ - mag variances noted above are reflective of the small discrepancies that can emerge from the different methods in ways that are often still hard to pinpoint and that are not even consistent from one galaxy to another. For a more extensive discussion on another system (NGC 5128) where several stellar candles can be accurately compared, see Harris et al. (2010). 


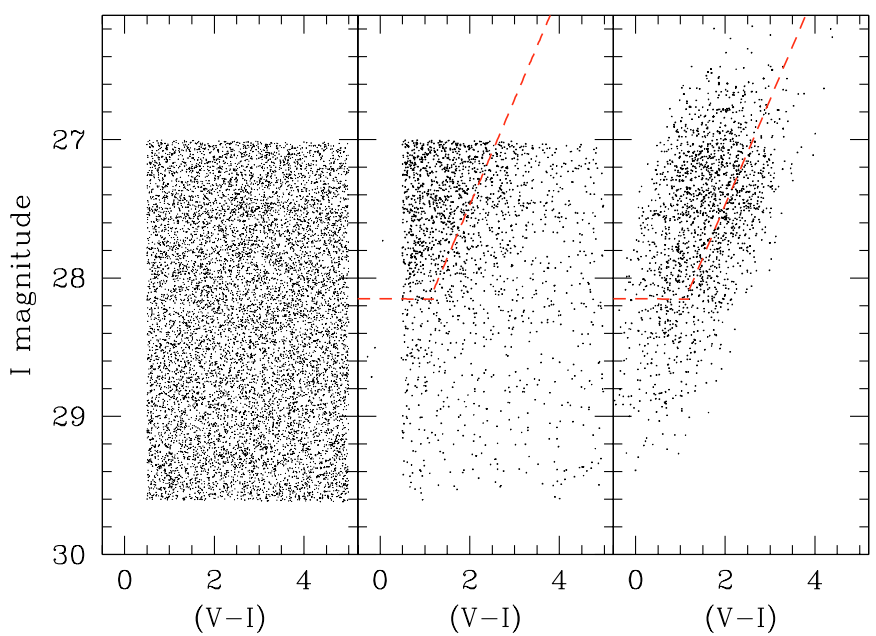

Fig. 7. Results of the first artificial-star set of tests. Left panel shows the color-magnitude distribution of the artificial stars inserted into the region of the image described in the text. Middle panel shows the stars from the left panel that were actually found and measured on both frames. Finally, the right panel shows the measured photometry for the stars that were recovered. The heavy dashed lines in the middle and right panels show the 50\% detection completeness levels.

Another direct, though somewhat less precise, comparison method of interest is the linear size distribution of globular clusters (GCs), which has been developed by Jordán et al. (2005) into a standard-ruler technique. The key quantity is the peak of the GC half-light radius distribution, normalized to host galaxy size and calibrated via the Milky Way GCs. Using the Jordán et al. data for M 87 and their calibration, we obtain $d=(16.4 \pm$ 2.3) $\mathrm{Mpc}$ or $(m-M)_{0}=31.07 \pm 0.30$.

Combining the four methods listed above (TRGB, PNLF, GC sizes, SBF/Cepheids), we obtain a weighted average distance modulus $\langle m-M\rangle_{0}=31.08 \pm 0.06$ for $\mathrm{M} 87$, or $d=$ $(16.4 \pm 0.5) \mathrm{Mpc}$. A more precise TRGB distance especially could be obtained very straightforwardly with halo-star photometry in a less crowded region, and would in our view be the most effective way to calibrate the distance to this important galaxy.

\section{Tests of the photometry}

To test the internal errors and completeness of the photometry we ran two separate artificial-star procedures. In the first series, stars were added to a representative $1500 \times 500$-px region of the image in the lower left corner (similar to the region shown in Fig. 1). These added stars were distributed evenly in color and magnitude over the ranges $0.5<(V-I)<5.0$ and $27.0<I<29.6$, as shown in Fig. 7. These intervals deliberately covered a larger range in both color and magnitude than in our observed CMD (Fig. 3). The same daophot measurement sequence as done on the original frames was then carried out, with a two-pass sequence of find/phot/allstar.

Figure 7 shows the results for inserted stars that were actually recovered in the photometry, including both their input magnitudes and colors (center panel) and their actually measured values (right panel). Of the total of 10000 added stars, just 1917 of these were successfully found and measured in both colors, and a high fraction of these lie in the upper left (bright, blue) part of the CMD. The completeness of detection $f=n$ (recovered) $/ n$ (input) as a function of magnitude is shown in Fig. 8; the 50\% completeness levels are reached at $F 606 W=29.00$ and $F 814 W=28.15$. To be classified as "recovered" a star must be detected in both

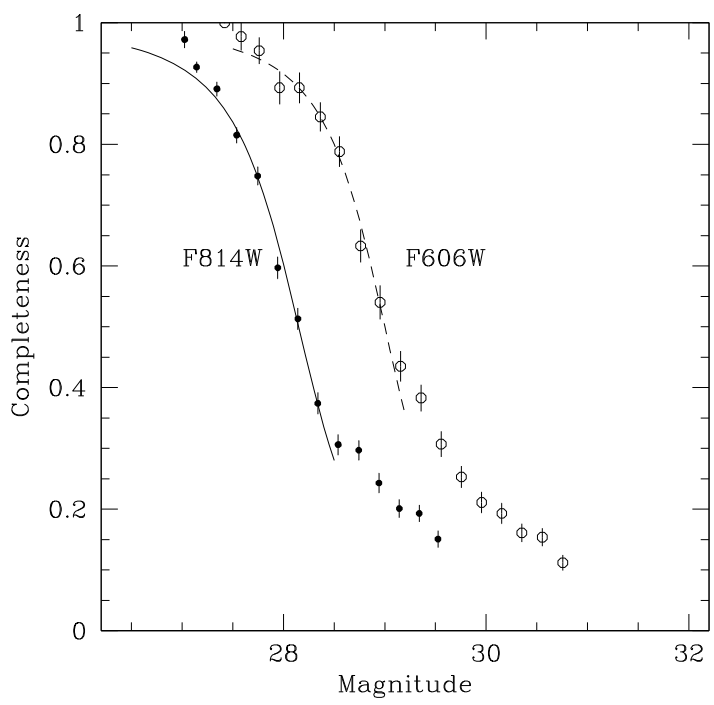

Fig. 8. The photometric completeness as determined from the artificialstar tests in the previous figure. The ratio $f$ represents the fraction of input stars actually found and measured in both colors, and is plotted here as a function of magnitude in each filter. The curves drawn through the brighter parts of each set of points is a standard interpolation function of the form $f=\frac{1}{2}\left[1-\alpha\left(m-m_{0}\right) / \sqrt{1+\alpha^{2}\left(m-m_{0}\right)^{2}}\right]$ where $m_{0}$ is the $50 \%$ completeness level and $\alpha$ governs the steepness of the falloff.

images. Note that at very faint levels (below the $50 \%$ point) the formal values of $f$ tend to decrease rather slowly, a result of the very high degree of crowding.

Figure 7 (particularly the difference between the second and third panels) clearly indicates that internal random uncertainties are large at all levels of the CMD. Figure 9 displays the differences between the measured and input magnitudes more completely. For $I \gtrsim 28.0$, one magnitude below the RGB tip, completeness of detection becomes low and the systematic errors increase, such that most stars are measured too bright. We do not consider this faint region further. The measured colors are on average slightly too blue independent of magnitude, by $\langle\Delta(V-I)\rangle=-0.22$ mag. Over the top magnitude of the RGB $(I=27-28)$ the internal random scatter of the magnitudes and colors (rms) is $\sigma= \pm 0.36 \mathrm{mag}$ in $F 814 W, \pm 0.53 \mathrm{mag}$ in $F 606 \mathrm{~W}$, and $\pm 0.40 \mathrm{mag}$ in $(F 606 W-F 814 W)$.

The artificially even distribution of input stars in Fig. 7 overpopulates the brightest part of the luminosity function compared with the real CMD. A second run of artificial-star testing was therefore done, this time by inserting a set of RGB stars that followed a real CMD for the halo of a giant elliptical. We took the photometry for the halo stars of NGC 5128 measured by Rejkuba et al. (2005) with deep HST/ACS exposures that reach the horizontal branch and thus accurately populate the entire luminosity and color range of interest here. Of the more than 77000 stars in the Rejkuba et al. dataset, about half (38640) were chosen randomly; these were shifted to the distance and reddening of M 87 to ensure that they occupied the same range in intrinsic luminosity and color; and were assigned random $(x, y)$ locations in the same $1500 \times 500$ section of the M 87 image as before. These added stars cloned from NGC 5128 increased the total light in the image section by less than $2 \%$.

The results of the remeasurement process are shown in Fig. 10. Of 5200 input stars brighter than $I=29.8$, just 570 were recovered after the 2-pass photometry. Interestingly, the faintest ones recovered $(I \gtrsim 29)$ often have very large systematic errors that push them well upward in the CMD. However, the region 
S. Bird et al.: The inner halo of M 87: a first direct view of the red-giant population

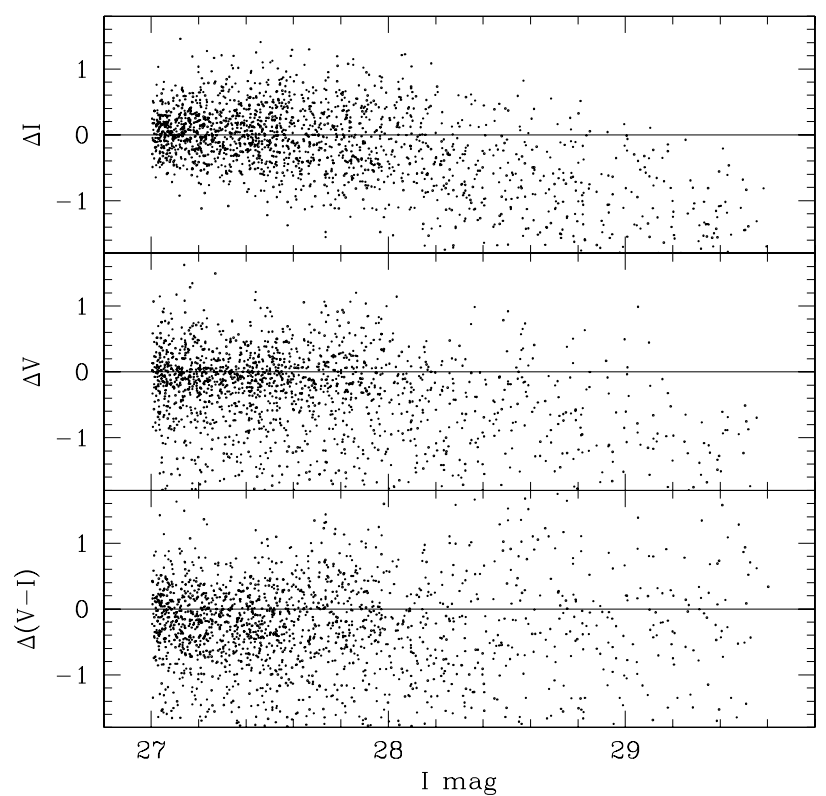

Fig. 9. Random uncertainties in the photometry, determined from the first series of artificial-star tests described in the text. The first and second panels show the differences $\Delta I, \Delta V$ (measured minus input) for the recovered stars, while the third panel shows the difference in color.

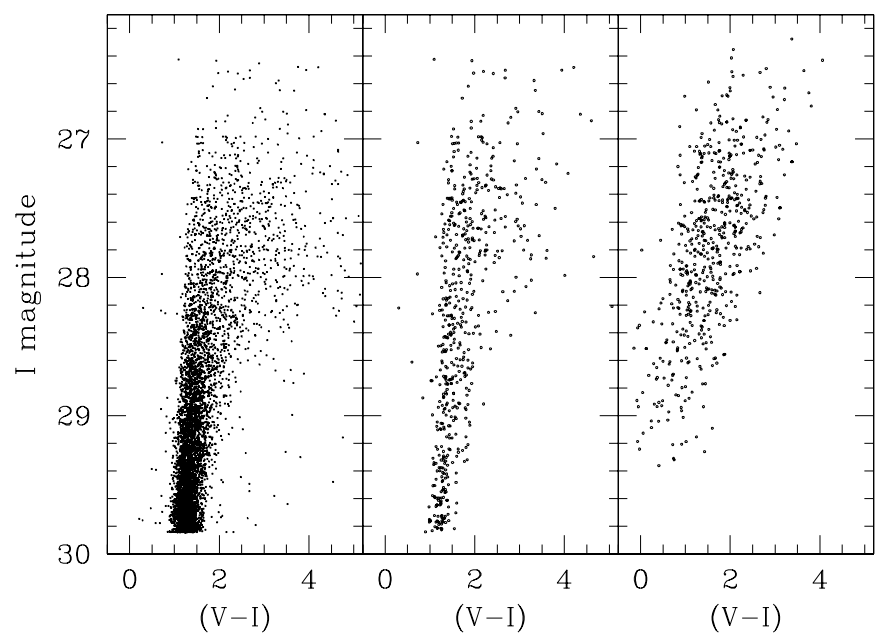

Fig. 10. Results of the second artificial-star set of tests. Left panel shows the color-magnitude distribution of the artificial stars inserted into the image section as described in Sect. 5. The stars are taken from the NGC 5128 halo field measured by Rejkuba et al. (2005), shifted to the distance and reddening of M 87. Middle panel shows the stars from the left panel that were actually found and measured on both frames; and the right panel shows the measured photometry for the stars that were recovered.

$I<28$ preserves much useful structure representing the metallicity range, losing only the reddest part of the range.

\section{The metallicity distribution}

The tests of the photometry described above lead us to restrict the MDF analysis to the brightest part of the RGB at $28.0>I>$ 27.0. In this range, even the random \pm 0.40 -mag scatter in $(V-I)$ is much smaller than the 1.5 -mag intrinsic spread in the actual $\mathrm{CMD}$, enough to give us workable leverage on the underlying metallicity distribution. In addition, the $\simeq 0$.2-mag systematic error in the colors is far smaller than the intrinsic color range of

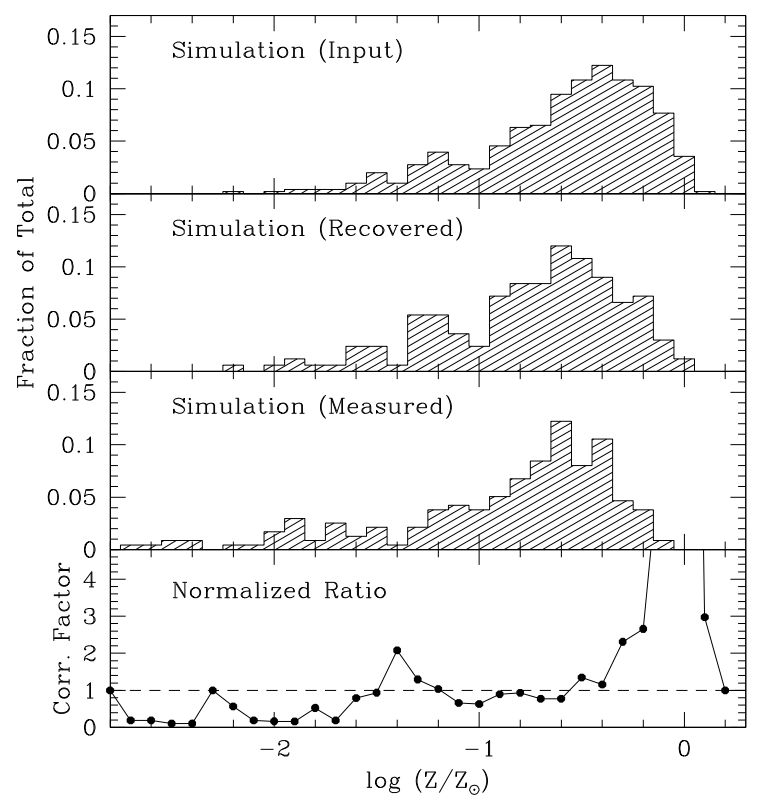

Fig. 11. Calibration tests of the metallicity distribution. In the top panel, the MDF is shown for the all the NGC 5128 stars from $I=27$ to 28 from the first panel of the previous figure. In the second panel, the MDF is shown for all the NGC 5128 stars that were recovered in the simulation tests (those in the middle panel of the previous figure). In the third panel, the MDF for these same recovered stars is shown, but now derived from their photometry as actually measured from the simulations (i.e. from the third panel in the previous figure). All three panels are normalized to the same number of stars. The bottom panel shows the ratio of the first and third histograms, normalized to an overall average of 1.00.

the red-giant tracks as they fan out toward the top of the RGB (Fig. 4), creating a shift at worst of $0.1-0.2$ dex in mean metallicity.

To estimate the combined effects of the quality of photometry on the deduced MDF, we used the NGC 5128 data (Fig. 10) again as a testbed. With the procedures described in detail in previous papers (Harris et al. 2002, 2007b; Rejkuba et al. 2005), interpolation within the grid of RGB tracks yields the MDF in the form of number of stars per unit interval in $[\mathrm{m} / \mathrm{H}]=\log \left(Z / Z_{\odot}\right)$. The inner halo stars within $8 \mathrm{kpc}$ in NGC 5128 are now well established to have a broad, predominantly metal-rich MDF peaking near $[m / H] \simeq-0.3$, and other giant ellipticals are expected to be similar (see the extensive discussions in Harris et al. 2002; Rejkuba et al. 2005 among others).

The RGB-grid interpolation procedure applied to the NGC 5128 stars is shown in three different versions in Fig. 11. In the top panel, we take the 507 stars in Fig. 10a that lie in our adopted range $27<I<28$ and derive the MDF in histogram form as shown there. This can be thought of as the intrinsic, input distribution free of photometric selection or scatter.

In the second panel, the MDF derived through the interpolation grid is shown for only the 167 NGC 5128 stars that were recovered in the simulation tests (that is, those in Fig. 10b). The $(I, V-I)$ values entered into the RGB grid are their true (input) values. The visible change in the MDF compared with the intrinsic version is the direct effect of the $V$-band cutoff, which is responsible for the loss of the highest-metallicity stars and thus also decreases the metallicity of the histogram peak.

In the third panel, the MDF is now shown for the recovered stars at $R_{\mathrm{gc}}=115^{\prime \prime}-155^{\prime \prime}$ or $9.3-12.5 \mathrm{kpc}$ between $I=27-28$ derived from their actually measured photometry (Fig. 10c). The overall histogram range and peak remain similar to the second 


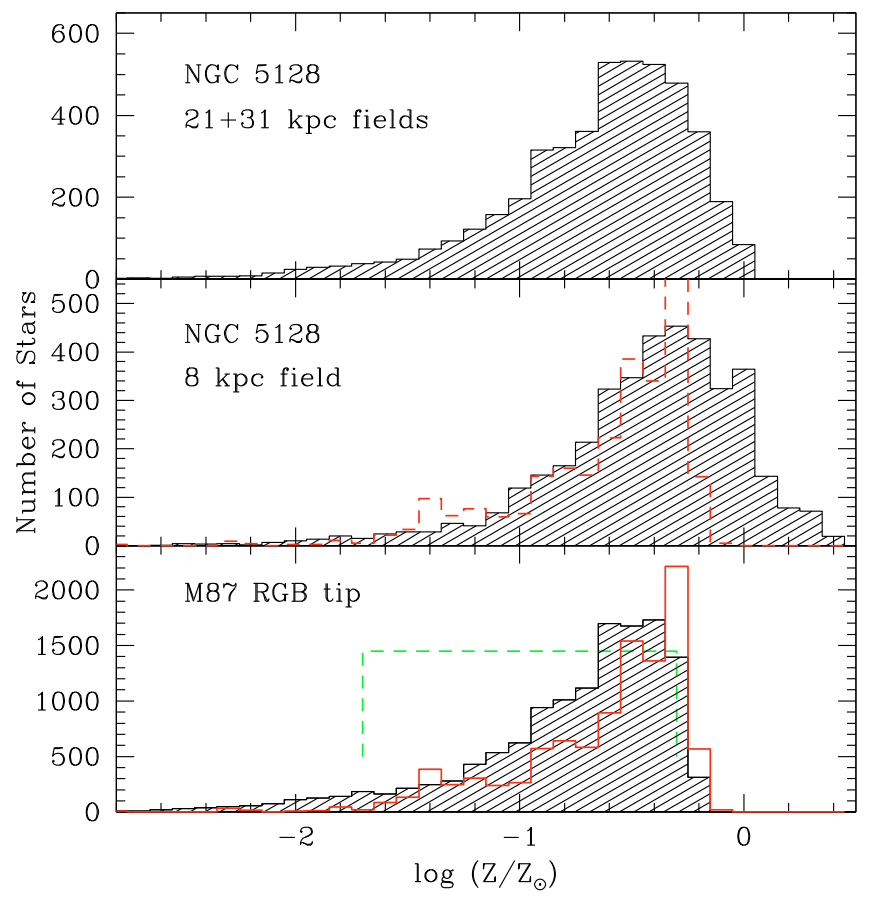

Fig. 12. Metallicity distribution for the halo stars in NGC 5128 and M 87. The upper two panels show the MDF (number of stars per 0.1dex metallicity interval) from three locations in the halo of NGC 5128 (see text). In the bottom panel, the shaded histogram shows the MDF for the stars in the M 87 inner halo in the magnitude range $I=27.0-28.0$, uncorrected for photometric completeness or color bias. The heavy red solid line shows the MDF now fully corrected for those effects, while the heavy green dashed line shows the metallicity range in which the deduced MDF is most reliable. This corrected MDF for M 87 is repeated in the middle panel (dashed line) for direct comparison with the inner-halo field in NGC 5128 (vertical scaling is arbitrary for display purposes).

panel, but relatively more stars appear at low metallicity because of the systematic bias of the colors toward the blue as mentioned above.

Lastly, the bottom panel of Fig. 11 shows the ratio of the first and third histograms (input versus measured), normalized to an overall average of 1.00 . We use this ratio to make a reasonable correction of the MDF for both the incompleteness (which affects mainly the high-metallicity end) and the color bias (which affects mainly the low-metallicity end).

The final results for our estimated MDF are shown in Fig. 12. The 13972 M 87 stars from Fig. 4 in the range $I=27.0-28.0$ as described above are put through the interpolation grid of RGB tracks to construct the raw MDF shown in the bottom panel (shaded histogram). This distribution is then multiplied by the correction factor shown in the bottom panel of Fig. 11 to produce the fully corrected $M D F$ shown as the heavy unshaded line. This corrected distribution represents our best estimate of the underlying MDF for the inner halo of M 87, corrected for photometric incompleteness and color measurement bias.

Even this corrected MDF can be relied on only for $[\mathrm{m} / \mathrm{H}] \lesssim$ -0.3 ; any stars more metal-rich than that are almost totally cut off from our data and thus no correction factors are useful. At the opposite end, the MDF shape for $[m / H] \lesssim-1.7$ is very poorly determined and we can say only that there are very few stars present in that range.

For direct comparison purposes we also show MDF data for the halo stars in three locations of the NGC 5128 halo, from
Harris et al. (1999, 2000, 2002). These were all taken with the HST WFPC2 camera in $(V, I)$ and have rather similar limiting absolute magnitudes to our M 87 ACS-based photometry, though they are less affected by crowding. The MDFs were derived in all cases with the same RGB grid of tracks and interpolation code. The mid-to-outer fields at projected galactocentric distances of 21 and $31 \mathrm{kpc}$ have MDFs that are virtually identical to each other and are combined in the upper panel of Fig. 12, while the inner-halo $8 \mathrm{kpc}$ field is shown by itself in the middle panel.

Our corrected MDF for the M 87 inner halo - at a mean projected distance of $10 \mathrm{kpc}$ - clearly resembles the 8 -kpc NGC 5128 field more closely than the outer fields. Over the range $[\mathrm{m} / \mathrm{H}]<-0.3$ where we can make the comparison, we conclude that the inner halos of both these giant ellipticals have basically similar MDFs that are broad, predominantly metal-rich, and with very small numbers of metal-poor stars. The MDF for M 87 may reach a peak near $[m / H] \sim-0.4$, but for the present this metallicity should be viewed as a lower limit to the peak value.

\section{Conclusions}

We have used HST Archive data for an unusually deep set of $F 606 W, F 814 W$ ACS images to probe the red-giant stellar population in the inner halo of M 87. Although the crowding levels of the faint halo stars in this field are severe at best, the regions from $115^{\prime \prime}-155^{\prime \prime}$ (9.3 to $12.5 \mathrm{kpc}$ projected galactocentric distance) give a useful first look at the brightest $1.5 \mathrm{mag}$ of the redgiant branch. This corresponds to distances of $1.4 R_{\mathrm{e}}-1.9 R_{\mathrm{e}}$, where $R_{\mathrm{e}}$ is the effective radius in the $I$ band of $81^{\prime \prime}(6.3 \mathrm{kpc})$ (Zeilinger 1993).

We have used this material to obtain a preliminary TRGBcalibrated distance to M 87 for the first time. We find a distance $d=(16.7 \pm 0.9) \mathrm{Mpc}$, in good agreement with the few other relatively direct methods available, including the planetary nebula luminosity function, the Cepheid-calibrated SBF method, and the linear diameters of globular clusters. Photometry of a more outlying halo field than the one we are able to study here has the potential to give a more precise TRGB determination and would represent the most direct possible distance calibration to the Virgo members at the present time.

As a second result of our study, we use the brightest 1 mag of the RGB stars to measure the metallicity distribution of the M 87 inner halo for the first time. We find that it is very broad and predominantly metal-rich, with a peak that may lie very near the completeness limit of our $V$-band data but is at least as metalrich as $[m / H] \simeq-0.4$. In general, the shape of the MDF strongly resembles the "8-kpc field" for the inner halo of NGC 5128, the nearby giant elliptical in the Centaurus group. In our view, these results strongly reinforce the view that the oldest stellar populations in giant E galaxies are fundamentally similar, regardless of whatever more recent accretion events may have happened to them.

Our study and the Williams et al. (2007) photometry of an intracluster Virgo field, show that there is considerable potential for extending this type of work to other Virgo members and the intracluster population as well. HST imaging with the ACS and WFC3 cameras is easily capable of resolving their halo redgiant populations and tracing the metallicity structures in this rich, nearby collection of large galaxies.

Acknowledgements. S.B. acknowledges the Wihurin Foundation and the Väsiälä Foundation for financial support. W.E.H. acknowledges support from the Natural Sciences and Engineering Research Council of Canada. 


\section{References}

Blakeslee, J. P., Lucey, J. R., Tonry, J. L., et al. 2002, MNRAS, 330, 443 Blakeslee, J. P., Anderson, K. R., Meurer, G. R., Benítez, N., \& Magee, D. 2003, Astronomical Data Analysis Software and Systems XII, ed. H. E. Payne, R. I. Jedrzejewski, \& R. N. Hook, ASP Conf. Ser. 295 (San Francisco, CA: ASP), 257

Blakeslee, J. P., Jordán, A., Mei, S., et al. 2009, ApJ, 694, 556

Caldwell, N. 2006, ApJ, 651, 822

Ciardullo, R., Jacoby, G. H., Feldmeier, J. J., \& Bartlett, R. E. 1998, ApJ, 492, 62

Freedman, W. L., Madore, B. F., Gibson, B. K., et al. 2001, ApJ, 553, 47

Gregg, M. D., Ferguson, H. C., Minniti, D., et al. 2004, AJ, 127, 1441

Harris, G. L. H., Harris, W. E., \& Poole, G. B. 1999, AJ, 117, 855

Harris, G. L. H., \& Harris, W. E. 2000, AJ, 120, 2423

Harris, G. L. H., Rejkuba, M., \& Harris, W. E. 2010, PASA, 27, 457

Harris, W. E., \& Harris, G. L. H. 2002, AJ, 123, 3108

Harris, W. E., Durrell, P. R., Pierce, M. J., \& Secker, J. 1998, Nature, 395, 45

Harris, W. E., Harris, G. L. H., Layden, A. C., \& Stetson, P. B. 2007a, AJ, 134, 43

Harris, W. E., Harris, G. L. H., Layden, A. C., \& Wehner, E. M. H. 2007b, ApJ, 666,903

Jordán, A., Côté, Patrick, B., John P., et al. 2005, ApJ, 634, 1002

Kormendy, J., Fisher, D. B., Cornell, M. E., \& Bender, R. 2009, ApJS, 182, 216

Madrid, J. P., Harris, W. E., Blakeslee, J. P., \& Gómez, M. 2009, ApJ, 705, 237

McConnachie, A. W., Irwin, M. J., Ferguson, A. M. N., et al. 2004, MNRAS, 350,243
McConnachie, A. W., Irwin, M. J., Ferguson, A. M. N., et al. 2005, MNRAS, 356,979

Mei, S., Blakeslee, J. P., Côté, P., Mei, S., et al. 2007, ApJ, 655, 144

Mouhcine, M., Ferguson, H. C., Rich, R. M., Brown, T. M., \& Smith, T. E. 2005a, ApJ, 633, 810

Mouhcine, M., Ferguson, H. C., Rich, R. M., Brown, T. M., \& Smith, T. E. 2005b, ApJ, 633, 821

Mouhcine, M., Rejkuba, M., \& Ibata, R. 2007, MNRAS, 381, 873

Peng, E. W., Jordán, A., Blakeslee, J. P., et al. 2009, ApJ, 703, 42

Pritchet, C., \& van den Bergh, S. 1985, ApJ, 288, L41

Rejkuba, M. 2004, A\&A, 413, 903

Rejkuba, M., Greggio, L., Harris, W. E., Harris, G. L. H., \& Peng, E. W. 2005, ApJ, 631, 262

Renzini, A. 2006, ARA\&A, 44, 141

Rizzi, L., Tully, R. B., Makarov, D., Makarova, L., et al. 2007, ApJ, 661, 815

Sirianni, M., Jee, M. J., Benítez, N., Sirianni, M., et al. 2005, PASP, 117, 1049

Stetson, P. B. 1987, PASP, 99, 191

Thomas, D., Maraston, C., Bender, R., \& Mendes de Oliviera, C. 2005, ApJ, 621, 673

Tonry, J. L., Dressler, A., Blakeslee, J. P., Tonry, J. L., et al. 2001, ApJ, 546, 681 VandenBerg, D. A., Swenson, F. J., Rogers, F. J., Iglesias, C. A., \& Alexander, D. R. 2000, ApJ, 532, 430

Waters, C. Z., Zepf, S. E., Lauer, T. R., \& Baltz, E. A. 2009, ApJ, 693, 463

Williams, B. F., Ciardullo, R., Durrell, P. R., et al. 2007, ApJ, 656, 756

Zeilinger, W. W., Møller, P., \& Stiavelli, M. 1993, MNRAS, 261, 175 